\title{
INTERSECTIONS OF COMMUTANTS OF ANALYTIC TOEPLITZ OPERATORS
}

\author{
JAMES E. THOMSON ${ }^{1}$
}

ABSTRACT. In this paper we study the intersection of commutants of analytic Toeplitz operators. Our main result is that if $\phi$ is a finite Blaschke product and $\Psi \in H^{\infty}$, then $\left\{T_{\phi}\right\}^{\prime} \cap\left\{T_{\Psi}\right\}^{\prime}=\left\{T_{l}\right\}^{\prime}$ where $I$ is a finite Blaschke product and $\phi$ and $\boldsymbol{\Psi}$ are functions of $I$. The key step is a function-theoretic theorem describing the relationship between a finite Blaschke product and any $H^{\infty}$ function.

1. Introduction. Let $H^{2}$ denote the Hilbert space of functions $f$ analytic in the open unit disk $D$ which satisfy $\sup _{0 \leq r<1} \int\left|f\left(r e^{i \theta}\right)\right|^{2} d \theta<\infty$. Let $H^{\infty}$ denote the algebra of bounded analytic functions on $D$. For $\phi \in H^{\infty}, T_{\phi}$ is the analytic Toeplitz operator on $H^{2}$ defined by $T_{\phi} f=\phi f$. The study of analytic Toeplitz operators has been extensive and many of their properties are well known [1] [3]. The commutant of analytic Toeplitz operators has recently been studied by Deddens and Wong [2]. Their main result is a sufficient condition for the commutant of an analytic Toeplitz operator to be the intersection of the commutants of the analyt ic Toeplitz operators corresponding to the inner and outer parts of the inducing $H^{\infty}$ function. The purpose of this paper is to study the intersection of commutants of analytic Toeplitz operators. In particular, we characterize the intersection of the commutant of an analytic Toeplitz operator induced by a finite Blaschke product with the commutant of any analytic Toeplitz operator. As a consequence, we obtain a strong partial answer to the last question raised by Deddens and Wong [2].

2. Notation and preliminaries. Let $\Gamma$ be the unit circle. If $f \in H^{2}$, then there is a function $\hat{f} \in L^{2}(\Gamma)$ such that $f\left(r e^{i \theta}\right)$ converges almost everyto $\hat{f}\left(e^{i \theta}\right) . \phi$ in $H^{\infty}$ is an inner function if $\left|\hat{\phi}\left(e^{i \theta}\right)\right|=1$ almost everywhere. $F$ in $H^{\infty}$ is outer if $T_{F}$ has dense range. Every $f$ in $H^{\infty}$ has a factorization $f=\phi F$ where $\phi$ is inner and $F$ is outer [4].

An isometry $T$ on $H^{2}$ is a pure isometry if $\bigcap_{n=0}^{\infty} T^{n} H^{2}=\{0\}$. For $f \epsilon$ $H^{\infty}, T_{f}$ is a pure isometry if and only if $f$ is a nonconstant inner function.

Received by the editors June 18, 1974 .

AMS (MOS) subject classifications (1970). Primary 47B35, 47B20, 30 A76.

Key words and phrases. Analytic function, inner function, $H^{\infty}, H^{2}$, analytic Toeplitz operator, commutant.

1 This paper represents part of the author's doctoral dissertation written at the University of North Carolina under the direction of Professor Joseph A. Cima. 
Operator will mean a bounded linear map from $H^{2}$ into $H^{2}$. For an operator $T$, let $\{T\}^{\prime}$ denote the commutant of $T$, i. e. the set of operators which commute with $T .\{T\}^{\prime}$ is a weakly closed subalgebra of the algebra of operators. It is important to note that there is a characterization of $\{T\}^{\prime}$ when $T$ is a pure isometry [2]. Hence, it is quite useful to reduce a problem to that of finding $\{T\}^{\prime}$ for $f$ an inner function.

$[X, Y, \ldots]$ will mean the closed linear span of the vectors $X, Y, \ldots$, and $\bar{a}$ will denote the complex conjugate of $a$. Two functions which we will need are $K_{z}(\lambda)=(1-\bar{z} \lambda)^{-1}$ and $B_{z}(\lambda)=(z-\lambda)(1-\bar{z} \lambda)^{-1}$. For notational convenience, let

$$
\phi_{z}(\lambda)=(\phi(z)-\phi(\lambda))(1-\overline{\phi(z)} \phi(\lambda))^{-1} \text {. }
$$

Also, let $(\cdot,$.$) denote the inner product on H^{2}$.

3. Main results. The main results of this paper are motivated by the following question raised in [2]. Suppose $\left\{J_{a}\right\}_{a \in A}$ is a family of inner functions. Is $\bigcap_{a \in A}\left\{T_{J_{a}}\right\}^{\prime}=\left\{T_{I}\right\}^{\prime}$ where $I$ is some inner function and each $J_{a}$ is a function of $I$ ? Our results support the idea that inner functions play a significant role in the study of the commutants of analytic Toeplitz operators. Theorem 1 is purely function theoretic, but it is the basis for the proof of Theorem 2, which is the key operator theoretic result. First, we present a function theoretic lemma.

Lemma. Suppose $R$ is a rational function with poles off the closed unit disk. Let $A=\{z \in D: R-R(z)$ has no multiple zeros $\}$. Then $D-A$ is a finite set.

Proof. Let $B=\left\{z: R^{\prime}(z)=0\right\}$. It is easy to see that $R^{-1}(R(B))=D-A$. Since $R$ is a rational function, we have that $D-A$ is a finite set.

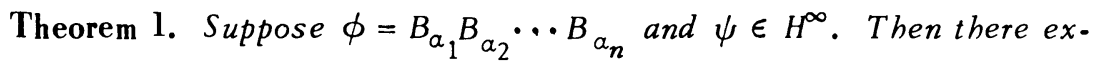
ist $m$ dividing $n$ and $C$ an open subset of $D$ with $D-C$ countable such that for each $z$ in $C, \phi^{-1}(\phi(z)) \cap \psi^{-1}(\psi(z))$ is a set containing precisely $m$ points. Moreover, there exists a finite Blaschke product, $I$, with $m$ factors such that $\phi$ and $\psi$ are functions of $I$.

Proof. Let

$E=\left\{z \in D: \phi_{z}\right.$ has no multiple zeros $\}=\left\{z \in D: \phi^{-1}(\phi(z))\right.$ has $n$ points $\}$

By the lemma $D-E$ is a finite set. For some $w \in E$, the zeros of $\phi_{w}$ are distinct and $\phi_{w}(0) \neq 0$. Since $\phi_{w}=B_{\phi(w)}{ }^{\circ} \phi$ and $\phi_{w}^{-1}\left(\phi_{w}(z)\right)=\phi_{w}^{-1}(\phi(z))$, we can assume that the $\alpha_{i}$ 's are distinct and nonzero.

For $j=1,2, \ldots, n$, let 
$E_{j}=\left\{z \in E: \phi^{-1}(\phi(z)) \cap \psi^{-1}(\psi(z))\right.$ has precisely $j$ points $\}$.

Let $E_{j}^{\prime}$ be the set of isolated points of $E_{j}$, and let $E_{j}^{\prime \prime}=E_{j}-E_{j}^{\prime}$. Note that $E=\bigcup_{j=1}^{n} E_{j}$

First, we claim that $E_{j}^{\prime \prime}$ is open. Fix $z_{0} \in E_{j}^{\prime \prime}$. There exists an open set $U$ containing $\phi\left(z_{0}\right)$ such that $\phi^{-1}(U)=\bigcup_{i=1}^{n} U_{i}$ where $\left\{U_{i}\right\}_{i=1}^{n}$ is a pairwise disjoint collection of connected, open sets with $\phi\left(U_{i}\right)=U$. Let $g_{i}=\left.\phi\right|_{U_{i}}$ and let $a_{i}=g_{i}^{-1} \circ g_{1}$. For $z \in U_{1}, \phi^{-1}(\phi(z))=\left\{a_{i}(z)\right\}_{i=1}^{n}$. Since $z_{0} \in E_{j}, \phi^{-1}\left(\phi\left(z_{0}\right)\right) \cap \psi^{-1}\left(\psi\left(z_{0}\right)\right)=\left\{a_{i}\left(z_{0}\right)\right\}_{i=1}^{j}$ (possibly reordering the $a_{i}$ 's). Hence, $\psi\left(a_{i}\left(z_{0}\right)\right) \neq \psi\left(z_{0}\right)$ for $i>j$, and thus, by the continuity of $\psi$ and the $a_{i}^{\prime}$ s, $\psi\left(a_{i}(z)\right) \neq \psi(z)$ for $i>j$ and $z$ near $z_{0}$. We can therefore assume that $\psi\left(a_{i}(z)\right) \neq \psi(z)$ for $i>j$ and $z \in U_{1}$ (choosing a smaller $U$, if necessary).

Since $z_{0} \in E_{j}^{\prime \prime}$, there exists $\left\{z_{k}\right\}_{k=1}^{\infty} \subseteq E_{j} \cap U_{1}$ such that $z_{k} \rightarrow z_{0}$. By the above, $\psi\left(a_{i}\left(z_{k}\right)\right) \neq \psi\left(z_{k}\right)$ for $i>j$. Hence, it must be the case that $\psi\left(a_{i}\left(z_{k}\right)\right)=\psi\left(z_{k}\right)$ for $i \leq j$. Therefore, $\psi\left(a_{i}(z)\right)=\psi(z)$ for all $z$ in $U_{1}$ and $i \leq j$. Consequently, $\phi^{-1}(\phi(z)) \cap \psi^{-1}(\psi(z))=\left\{a_{i}(z)\right\}_{i=1}^{j}$ for all $z$ in $U_{1}$, and thus, $U_{1}$ is contained in $E_{j}$. Hence, $E_{j}^{\prime \prime}$ is open and the claim is established.

Let $A=(D-E) \cup\left(\bigcup_{j=1}^{n} E_{j}^{\prime}\right)$. Note that $A$ is countable, $D-A$ is a connected, open set, and $D-A=\bigcup_{j=1}^{n} E_{j}^{\prime \prime}$. Since $D-A$ is connected and $\left\{E_{j}^{\prime \prime}\right\}$ is a pairwise disjoint collection of open sets, $D-A=E_{m}^{\prime \prime}$ for some $m$. Let $C=E_{m}^{\prime \prime}$. Take $z$ such that $\phi^{-1}(\phi(z)) \subseteq C$. Note that

$$
\phi^{-1}(\phi(z))=\left\{a_{i}(z)\right\}_{i=1}^{n} \text { and } \phi^{-1}\left(\phi\left(a_{i}(z)\right)\right)=\left\{a_{j}(z)\right\}_{j=1}^{n} \text {. }
$$

We know $\phi^{-1}(\phi(z)) \cap \psi^{-1}(\psi(z))$ has $m$ points, so we can assume $\phi^{-1}(\phi(z))$ $\cap \psi^{-1}(\psi(z))=\left\{a_{i}(z)\right\}_{i=1}^{m}$. Since $a_{m+1}(z) \in C, \phi^{-1}\left(\phi\left(a_{m+1}(z)\right)\right) \cap$ $\psi^{-1}\left(\psi\left(a_{m+1}(z)\right)\right)$ has $m$ points and we can assume that they are $\left\{a_{i}(z)\right\}_{i=m+1}^{2 m}$. If we continue this process, we eventually exhaust $\phi^{-1}(\phi(z))$. This gives us $\phi^{-1}(\phi(z))$ as a disjoint union of sets with $m$ points each. Hence $m$ divides $n$. We have now proven the first conclusion of the theorem and we will proceed to the proof of the second conclusion. Note that the fact that $m$ divides $n$ is also an immediate consequence of the second conclusion.

For $z \in C$, let $I(z)$ be the product of the $m$ numbers in $\phi^{-1}(\phi(z)) \cap$ $\psi^{-1}(\psi(z))$ and let $J(z)$ be the product of the other $n-m$ numbers in $\phi^{-1}(\phi(z))$. For $z \in E$, let $L(z)$ be the product of the $n$ numbers in $\phi^{-1}(\phi(z))$. We are now going to show that $I(z)$ can be extended to a finite Blaschke product.

Fix $z_{0} \in C$. We have shown that there exists $\left\{a_{i}\right\}_{i=1}^{n}$ with each $a_{i}$ defined and analytic in a neighborhood of $z_{0}$ such that $\phi^{-1}(\phi(z)) \cap \psi^{-1}(\psi(z))$ $=\left\{a_{i}(z)\right\}_{i=1}^{m}$ for all $z$ near $z_{0}$. Hence $I(z)=\prod_{i=1}^{m} a_{i}(z)$ for all $z$ near $z_{0}$. 
Therefore, $I$ is analytic at $z_{0}$. Since $z_{0}$ was an arbitrary point in $C, I$ is analytic on $C$. Combining this with the facts that $D-C$ is countable and $I$ is bounded by one in modulus, $I$ can be extended analytically to $D$ and the extension is in $H^{\infty}$. We identify $I$ with its extension.

Next, we claim that $I$ is, in fact, a finite Blaschke product. Given $\epsilon>$ 0 there exists $\delta>0$ such that $|z|>1-\delta$ and $z \in C$ implies $|I(z)|>1-\epsilon$. Since $I$ is bounded by one in modulus, $|I(z)| \rightarrow 1$ uniformly as $|z| \rightarrow 1$. Therefore, $I$ is a finite Blaschke product. By arguments similar to the above, one can show that $J$ and $L$ can also be extended to finite Blaschke products. Clearly, $L=1 J$. Note that $z \in \phi^{-1}(\phi(z))$ so $a_{i}(z)=z$ for some $i$. Therefore, $|L(z)| \leq|z|$ and hence, $L(0)=0$.

Since

$$
L_{\alpha_{1}}=\frac{L\left(\alpha_{1}\right)-L}{1-\overline{L\left(\alpha_{1}\right) L}}
$$

we have

$$
L_{a_{1}}^{-1}(0)=L^{-1}\left(L\left(\alpha_{1}\right)\right) \supseteq\left\{\alpha_{i}\right\}_{i=1}^{n}
$$

(recalling that the $a_{i}$ 's are the $n$ distinct zeros of $\phi$ ). Therefore, $\phi$ divides $L_{a_{1}}$. Also, $\phi(0)=a_{1} \alpha_{2} \cdots a_{n}$ and

$$
L_{a_{1}}(0)=\frac{L\left(\alpha_{1}\right)-L(0)}{1-L\left(a_{1}\right) L(0)}=L\left(a_{1}\right)=a_{1} a_{2} \cdots a_{n}
$$

by the definition of $L$. Since $\phi$ divides $L_{\alpha_{1}}$ and $\phi(0)=L_{a_{1}}(0) \neq 0, \phi=L_{\alpha_{1}}$. Therefore, $L$ is a finite Blaschke product with $n$ factors.

Take any $z \in C$. Then $I^{-1}(I(z)) \supseteq\left\{a_{i}(z)\right\}_{i=1}^{m}$ and $J^{-1}(J(z)) \supseteq$ $\left\{a_{i}(z)\right\}_{i=m+1}^{n}$. Therefore, $I$ has at least $m$ Blaschke factors and $J$ has at least $n-m$ Blaschke factors. But $I J=L$ has exactly $n$ factors, so $I$ has precisely $m$ factors.

For $w \in I(C)$, let $F(w)=\phi \circ I^{-1}(w)$ and $G=\psi \circ I^{-1}(w)$. Since $I^{-1}(I(z))$ $=\phi^{-1}(\phi(z)) \cap \psi^{-1}(\psi(z))$ for all $z \in C, F$ and $G$ are well defined. It is easy to see that $F$ and $G$ can be extended analytically to $D$ and that $F \circ I$ $=\phi$ and $G \circ I=\psi$.

Theorem 2. Suppose $\phi$ is a finite Blaschke product and $\psi \in H^{\infty}$. Then $\left\{T_{\phi}\right\}^{\prime} \cap\left\{T_{\psi}\right\}^{\prime}=\left\{T_{I}\right\}^{\prime}$ where $I$ is a finite Blaschke product and $\phi$ and $\psi$ are functions of $I$.

Proof. Suppose $\phi$ has $n$ factors. Let $m, C$, and $I$ be those given by the last theorem. It is clear that $\left\{T_{\phi}\right\}^{\prime} \cap\left\{T_{\psi}\right\}^{\prime} \supseteq\left\{T_{I}\right\}^{\prime}$, so we only need to show the reverse inclusion. Let $\psi_{z}$ denote $\psi(z)-\psi$.

By the proof of the previous theorem, there exists $\left\{a_{i}\right\}_{i=1}^{n}$ with each 
$a_{i}$ analytic and nonvanishing on some open set $V$ contained in $C$ such that for $z \in V, \phi_{z}=a(z) B_{a_{1}(z)} \cdots B_{a_{n}(z)}$ and $\psi_{z}=B_{a_{1}(z)} \cdots B_{a_{m}(z)} F[z]$ where $|a(z)|=1$ and $F[z] \in H^{\infty}$ and does not vanish at $a_{i}(z)$ for $i>m$. We claim that for $z \in V$,

$$
\operatorname{ker} T_{\phi_{z}}^{*} \cap \operatorname{ker} T_{\psi_{z}}^{*}=\left[K_{a_{1}(z)}, \ldots, K_{a_{m}(z)}\right] \text {. }
$$

Fix $w \in V$. Let $b_{i}=a_{i}(w)$. Suppose $f \in \operatorname{ker} T_{\phi_{w}}^{*} \cap \operatorname{ker} T_{\psi_{w}}^{*}$. Then $f \epsilon$ $\operatorname{ker} T_{\phi_{w}}^{*}=\left[K_{b_{1}}, K_{b_{2}}, \ldots, K_{b_{n}}\right]$, so there exists $\left\{c_{i}\right\}_{i=1}^{n}$ such that $f=$ $\sum_{i=1}^{n} c_{i} K_{b_{i}}$. Let $G$ be the inner part of $\psi_{w}$ and let $H=B_{b_{m}+2} \cdots B_{b_{n}}$. Since $\left(G H, K_{b_{i}}\right)=G\left(b_{i}\right) H\left(b_{i}\right)=0$ for $i \neq m+1$, we have $(G H, f)=$ $c_{m+1} G\left(b_{m+1}\right) H\left(b_{m+1}\right)$. But $T_{\psi_{w}}^{*} f=0$ implies $T_{G}^{*} f=0$, so $\left(H, T_{G}^{*} f\right)=0$ or, equivalently, $(G H, f)=0$. Since $G\left(b_{m+1}\right) H\left(b_{m+1}\right) \neq 0, c_{m+1}=0$. By a similar argument we could show that $c_{i}=0$ for all $i>m+1$. Hence $f \epsilon$ $\left[K_{b_{1}}, \ldots, K_{b_{m}}\right]$. The reverse inclusion is obvious, so the proof of the claim is complete.

Suppose $T \in\left\{T_{\phi}\right\}^{\prime} \cap\left\{T_{\psi}\right\}^{\prime}$. Since $\left\{T_{\phi_{z}}\right\}^{\prime}=\left\{T_{\phi}\right\}^{\prime}$ and $\left\{T_{\psi_{z}}\right\}^{\prime}=\left\{T_{\psi}\right\}^{\prime}$, we have $T^{*} \in\left\{T_{\phi_{z}}^{*}\right\}^{\prime} \cap\left\{T_{\psi_{z}}^{*}\right\}^{\prime}$. Hence, $\operatorname{ker} T_{\phi_{z}}^{*} \cap \operatorname{ker} T_{\psi_{z}}^{*}$ is invariant under $T^{*}$. For $z \in V$, (1) holds, so there exist $F_{1}(z), F_{2}(z), \ldots, F_{m}(z)$ such that $T^{*} K_{z}=\overline{F_{1}(z)} K_{a_{1}(z)}+\cdots+\overline{F_{m}(z)} K_{a_{m}(z)}$. Hence, for $f \in H^{2}$ and $z \in V$,

$$
(T f)(z)=\left(T f, K_{z}\right)=\left(f, T^{*} K_{z}\right)=F_{1}(z) f\left(a_{1}(z)\right)+\cdots+F_{m}(z) f\left(a_{m}(z)\right) .
$$

Since $I\left(a_{i}(z)\right)=I(z)$ for $i \leq m,\left(T_{I} T f\right)(z)=\left(T T_{I} f\right)(z)$ for $z \in V$ and, hence, for all $z \in D$. Therefore $T \in\left\{T_{I}\right\}^{\prime}$ and the proof is complete.

Corollary 1. Let $\left\{J_{\alpha}\right\}_{a \in A}$ be a family of $H^{\infty}$ functions. Suppose that at least one of the $J_{\alpha}$ 's is a finite Blaschke product. Then $\bigcap_{a \in A}\left\{T_{J_{\alpha}}\right\}^{\prime}=$ $\left\{T_{I}\right\}^{\prime}$ where $I$ is a finite Blaschke product and each $J_{\alpha}$ is a function of $I$.

Proof. Let $J_{1}$ be a finite Blaschke product in the family $\left\{J_{a}\right\}_{a \in A}$. If $\left\{T_{J_{1}}\right\}^{\prime} \cap\left\{T_{J_{a}}\right\}^{\prime}=\left\{T_{J_{1}}\right\}^{\prime}$ for every $a \in A$, then we are finished by the theorem. Otherwise, there exists $\alpha_{1}$ such that $\left\{T_{J_{1}}\right\}^{\prime} \cap\left\{T_{J_{a_{1}}}\right\}^{\prime}=\left\{T_{J_{2}}\right\}^{\prime}$ where $J_{2}$ is a finite Blaschke product with fewer factors than $J_{1}$. If we continue this process, we will find a finite Blaschke product $I$ that works after a finite number of steps.

Corollary 2。 Suppose $\phi \in H^{\infty}$ has inner-outer factorization $\phi=B F$. If $B$ is a finite Blaschke product, then the following are equivalent:

(i) $\left\{T_{\phi}\right\}^{\prime}=\left\{T_{B}\right\}^{\prime} \cap\left\{T_{F}\right\}^{\prime}$;

(ii) $\left\{T_{\phi}\right\}^{\prime}=\left\{T_{I}\right\}^{\prime}$ where $I$ is a fin ite Blaschke product and $\phi$ is a function of $I$. 
Proof. (i) $\Rightarrow$ (ii). This is immediate from the theorem. (ii) $\Rightarrow$ (i). By (ii) there exists $\psi \in H^{\infty}$ such that $\phi=\psi \circ I$. Suppose $\psi=J G$ is the inner-outer factorization of $\psi$. Then $\phi=(J \circ I)(G \circ I)$. Since $J \circ I$ is inner and $G \circ I$ is outer, $B=a J \circ I$ and $F=b G \circ I$ where $|a|=|b|=1$. Hence, $\left\{T_{B}\right\}^{\prime} \cap\left\{T_{F}\right\}^{\prime} \supseteq\left\{T_{I}\right\}^{\prime}=\left\{T_{\phi}\right\}^{\prime}$. The reverse inclusion holds for any $\phi \in H^{\infty}$, so the proof is complete.

\section{REFERENCES}

1. A. Brown and P. R. Halmos, Algebraic properties of Toeplitz operators, J. Reine Angew. Math. 213 (1963/64), 89-102. MR 28 \#3350; 30, p. 1205.

2. J. Deddens and T. K. Wong, The commutant of analytic Toeplitz operators, Trans. Amer. Math. Soc. 184 (1973), 261-273.

3. R. G. Douglas, Banach algebra techniques in operator theory, Academic Press, New York, 1972.

4. P. L. Duren, Theory of $H^{p}$-spaces, Pure and Appl. Math., vol. 38, Academic Press, New York, 1970. MR 42 \#3552.

5. E. Nordgren, Reducing subspaces of analytic Toeplitz operators, Duke Math. J. 34 (1967), 175-181. MR $35 \# 7155$.

DEPARTMENT OF MATHEMATICS, UNIVERSITY OF NORTH CAROLINA, CHAPEL HILL, NORTH CAROLINA 27514

Current address: Department of Mathematics, Virginia Polytechnic Institute and State University, Blacksburg, Virginia 24061 